\title{
Raising Awareness of IoT Sensor Deployments
}

\author{
Mateusz Mikusz $z^{\dagger}$, Steven Houben ${ }^{\dagger}$, Nigel Davies ${ }^{\dagger}$, Klaus Moessner $^{\ddagger}$, Marc Langheinrich $^{\S}$ \\ ${ }^{\dagger}$ Lancaster University, ${ }^{\ddagger}$ University of Surrey, ${ }^{\S}$ Universit della Svizzera italiana \\ ${ }^{\dagger}\{$ m.mikusz, s.houben, n.a.davies $\} @$ lancaster.ac.uk, ${ }^{\ddagger}$ k.moessner@surrey.ac.uk, ${ }^{\S}$ marc.langheinrich@usi.ch
}

\begin{abstract}
The IoT is increasingly being used to support smart spaces and physical analytics and yet much of this smartness is made deliberately invisible to the user-echoing Weiser's vision of calm computing and technology that fades into the background. However, this means that users may not be aware or may not understand how the IoT is being deployed in their area. In other domains we know that a lack of awareness and a lack of understanding can lead to poor user experience/frustration, mistrust, suspicion, inability to capitalise on benefits and, security vulnerabilities. In this paper we present preliminary work that explores the issue of user awareness of IoT-based data collection.
\end{abstract}

Keywords: Internet of Things, Pervasive Displays, Sensors

\section{Introduction}

Rapid advances in low-cost sensing, actuation and communication technologies are leading to the wide-spread deployment of IoT devices. Such devices are often installed in public or semi-public spaces such as transport hubs, city streets, parks, public buildings, cafes and shared offices. These new IoT sensing devices and infrastructures can provide a wealth of data, enabling us to understand how spaces are used and to inform the organisation and management of available resources in urban areas [3]. The same sensors can also provide detailed insights into the behaviour of individuals present in spaces and can be used to support applications such as personal health monitoring, behaviour change, personalisation of spaces and detection of potential criminal activity. This type of data capture and use is often referred to as physical analytics [25].

However, in keeping with Weiser's vision of technology that fades into the background, much of the IoT technology that is being deployed is essentially designed to be invisible - the technology does not communicate its presence, purpose, practice and analysis to the wider audience it is monitoring. The result is that users of public spaces are increasingly unaware of the technology that is being used to capture physical analytics within the spaces they inhabit and, of how such analytics data is being exploited to provide new insights and new actionable outputs that directly affect people's lives.

In other domains we know that a lack of awareness and understanding lead to poor user experience and frustration, mistrust, suspicion, poor levels of adoption as a result of an inability to capitalise on benefits and, potential security vulnerabilities as a consequence of users' failure to understand the potential consequences of their actions.

A lack of awareness also leads to obvious concerns with respect to privacy and transparency and, additionally, results in a lack of opportunities for users to provide feedback [1, 15]. In particular, we note that a lack of awareness and engagement with those present in a physical space can reduce our ability to collect and interpret accurate IoT-based physical analytics data. Although modern analytical tools and machine learning approaches are useful to construct sophisticated data models, these are often still "sterile" and lack more qualitative insights. Because of the highly dynamic and volatile character of shared public spaces, it can be difficult to understand what changes in data really mean and how they can be leveraged to build better services or support emerging behaviour. The inclusion of "humans in the loop" when collecting analytics data offers the potential for users to explore these interpretations and provide qualitative user feedback that can be leveraged to increase the quality of data interpretations while making the collection process more transparent. Such inclusion of humans in the loop necessitates awareness of IoT infrastructure.

In this paper, we present preliminary work that explores the issue of user awareness of IoT based data collection for physical analytics. In particular, we report on the results of a focus group that explores user attitudes, a prototype that demonstrates the technical feasibility of using a pervasive display network to provide awareness of IoT sensing and we outline significant opportunities for new research in this space.

\section{Design Probe}

To explore user user attitudes towards awareness of IoT sensor data collection and visibility, we built the IoT Sensor Visualiser, a design probe that allows users to show IoT sensor data on nearby public or semi-public displays. Our design probe builds on top of existing IoT sensing and pervasive display technologies. The integration into existing real-world technologies allowed us to provide participants with a realistic design probe as it might appear in practise. 


\subsection{Underlying Technologies}

\subsubsection{IoT Sensor Network}

To collect real sensor readings in the user's context, we utilised the IoT Egg that is part of an "open-source, multimodal sensor suite" developed by the University of Surrey for a number of use cases including IoT test-beds, demonstration and "environmental contextualisation" [17]. Figure 3 shows the current version of the sensing device that consists of a number of built-in sensors and is capable of capturing ranging, particulate, noise, temperature, humidity, light and gesture. To provide feedback to the user, the egg is equipped with an LED and a vibration motor. Both the Wi-Fi and Bluetooth components can be used for configuration of the device and for real-time reporting of collected sensor data. We configured the IoT Eggs to report their sensor readings with a frequency of one reading per second to the "IoT Egg Cloud", a dedicated cloud service to which all IoT Eggs report using HTTP-based requests. Sensor readings are stored in a No-SQL database (MongoDB) and made available through a set of REST Application Programming Interfaces (APIs). The cloud service is managed and provided by the University of Surrey as part of an integrated IoT Hub. IoT Eggs are typically associated with a unique identifier that is used to retrieve the sensor readings from the cloud service.

\subsubsection{Display Network}

We utilise the open displays network at Lancaster as a test-bed for visualising and creating awareness of sensor readings. The display deployment is part of the world's largest research deployments in digital signage, and currently consists of over 65 pervasive displays located in student learning zones, the university library and at foyer areas of departmental buildings and student accommodations. The displays use the Yarely digital signage player [6] to retrieve content schedules and visualise content on displays. Typically, displays are configured to show a mix of content including departmental and college news and events mainly targeting visitors, students and members of staff. The content is usually composed of static images, videos or Web sites to visualise dynamic content. If Yarely tries to open a Web site, it includes a unique display identifier in the HTTP request header to enable the Web-based content to know which display is requesting the content. This can be used to, for example, determine the display location through a lookup table.

In addition to providing standard display content, the test-bed also supports the delivery of content tailored to viewers standing in the vicinity of a display [7]. In particular, users are able to download Tacita, a mobile phone application to configure their personal preferences regarding the types of content they would like to see across the display network. For example, users can choose from a set of applications such as weather or transportation information. The mobile phone application automatically monitors the user's proximity to displays. If the user is detected as being near to a display that supports the delivery of personalised content, the mobile phone application sends a request to the display network infrastructure to change the content on the display in accordance with the user's preferences. The display personalisation functionality supports the integration of new applications and content through a set of application programming interfaces.

\subsection{Application Design}

Our design probe's core function is to present sensor readings and recommendations to users through the public display network. The design probe dynamically shows the latest IoT sensor readings through simple graph visualisations. As shown in Figure 1 the initial design probe supports the visualisation of volume, dust, temperature and humidity values from the past four hours-allowing users to gain insights into both historic and current sensor values. In addition to the graph visualisations, the design probe also provides a set of recommendations to the user that are computed based on the sensor readings. In particular, the design probe uses current noise, humidity and temperature values to compute recommendations such as ensuring that the user maintains healthy temperature and humidity levels. For example, if the temperature is detected as being too high, an appropriate message is displayed to the user indicating that a certain action is required. Additionally, the transparent visualisation of both sensor readings and recommendations allows the user to quickly verify whether IoT sensors are reporting correct values or whether the sensor might be faulty.

The design probe also leverages our display personalisation technology to allow users to configure the IoT Egg used as a source of sensor readings. Through the Tacita mobile application, users can specify that they wish to see the design probe visualisation as their preferred piece of content as they walk by displays. In addition, the application allows users to specify the unique identifier of the specific IoT Egg to be used as the basis for the visualisation. Figure 2 shows the system architecture and its integration into the IoT Egg eco-system and open displays network.

The initial prototype of the design probe was implemented entirely in Python using the Tornado Web Framework and SQLite for caching sensor readings after requesting these from the cloud service. We deployed the prototype in the context of the Open Displays Network at Lancaster University and IoT Hub at Surrey.

\section{Exploration of User Attitudes}

To gain more insight and explore user attitudes toward IoT sensing devices that are collecting data in an environment, we conducted a focus group with six participants (4 female, 2 male).

\subsection{Design and Methodology}

We structured the focus groups into four parts: introduction and scene setting, discussion on sensing awareness and ways to access sensor data, and critique of the existing prototype system. We first set the scene with the participants - describing 


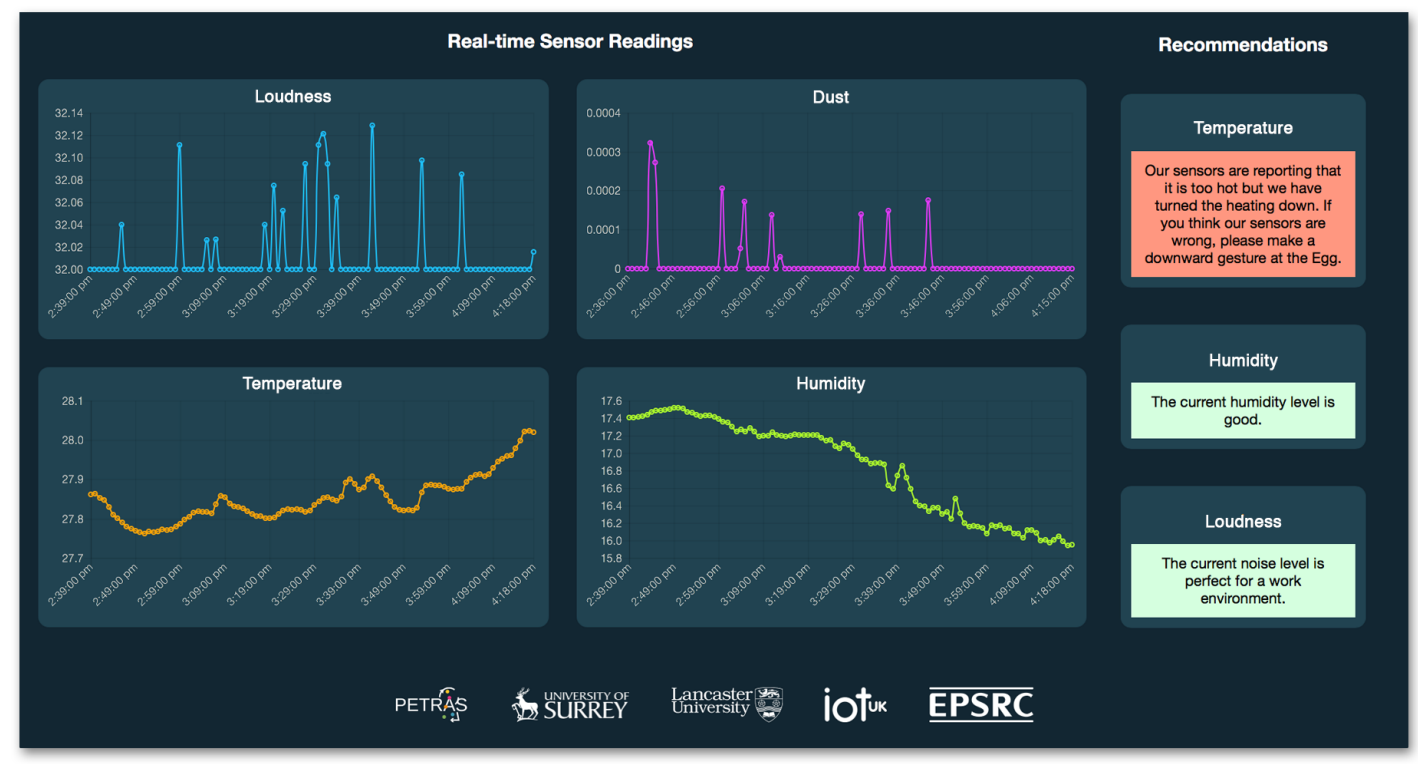

Fig. 1: Sensor visualisation to be shown on proximate public displays as users walk by.

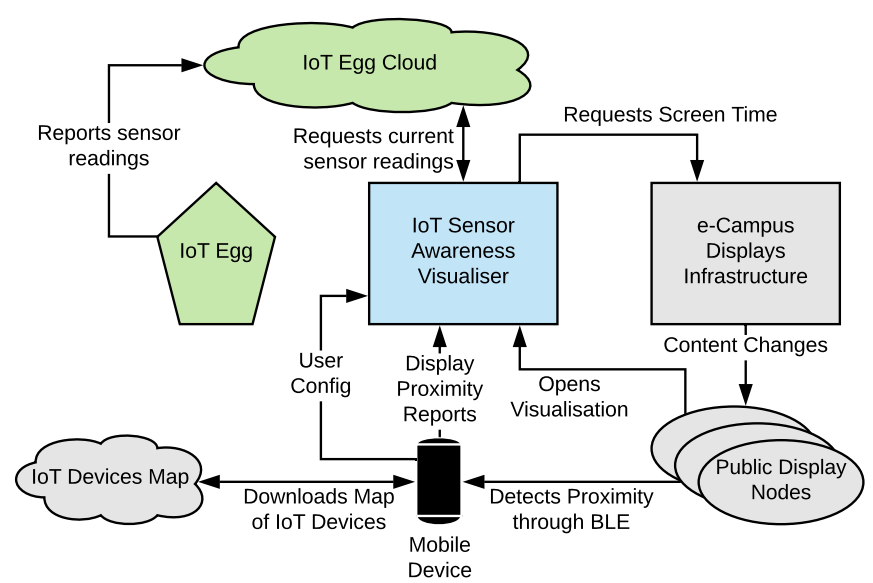

Fig. 2: Architecture and flow diagram for the design probe consisting of the IoT Egg infrastructure (green), open displays network (grey) and the design probe (blue).

the emergence of IoT sensing in the environment. To find out the participants' level of understanding with regards to sensing capabilities, we showed the participants the "IoT Egg" as a physical example of an IoT sensing device and asked participants to speculate on the possible sensors that might be built-in to the device. Having discussed sensing capabilities and given participants insights into the kinds of data sensors might collect, we initiated a brainstorming session on exploring ways to access and retrieve sensing data - and whether accessing such data would be important to participants. Finally, we showed participants our design probe, including the data visualisation and feedback techniques and asked for their views on the system. During the focus groups, we captured all ideas and concepts through notes, drawings and photographs of flip charts.

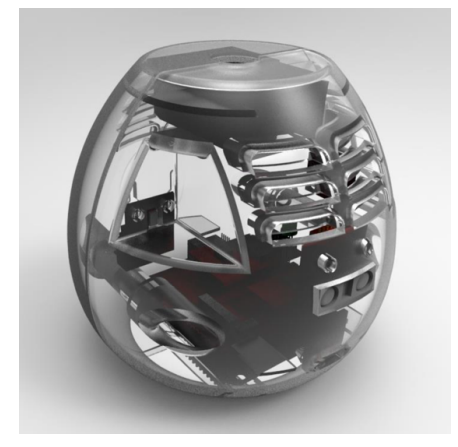

Fig. 3: The "IoT Egg" sensing device.

\subsection{Sensing Capabilities of the IoT Egg}

Participants generally struggled to identify sensing capabilities that might be built in to the IoT Egg or deployed in an environment. Sensors that were suggested included only a limited set of examples: "pressure pads," "motion sensors" and "movement sensors." Very quickly participants started to express concerns regarding audio and video recordings from the sensing device, though they were not able to recognise any concrete sensors within the the IoT Egg despite its transparent enclosure ("I don't see a camera or microphone.").

During the focus group participants commented on the physical characteristics of the IoT Egg as an IoT sensor device participants expected a non-transparent enclosure and smaller sized device (e.g. "I expected it to be closed, like an Amazon Echo." or "I expected it to be half the size."). For example, one participant noted that they would except a very small microphone or camera ("size of a finger nail") but not a large device. The transparent enclosure lead to interesting comments by participants, e.g. "see through and transparent makes me more curious." Participants though that transparency meant "nothing to hide" though they also recognised that they did not know 
what the IoT Egg did—even though it was transparent.

When asked about storage and usage of collected sensor data, participants were not able to identify any possible use cases. Furthermore, participants were rather naive on what could be identified from the collected dataset. For example, nonpersonally identifiable data (e.g. noise, energy, temperature) was considered as unimportant and participants were not worried about the collection of such data in both public and private spaces.

From this small sample set we believe that users have a very limited understanding of potential sensing capabilities and usage of collected datasets. The focus on the kinds of data that is collected (i.e. deployed sensor types) seemed to be more important than providing insights into the actual data that is collected.

\subsection{Awareness of IoT Sensing in the Environment}

Participants had concrete ideas on how users could be informed of sensing capabilities of an environment and the usage of data-however, participants noted that they differentiate between personally identifiable data (audio and video capture were mentioned as concrete examples) and other datasets. It was important to participants to be notified of data collection of personally identifiable data-even before entering a space with such sensing capabilities ("if it is identifiable then I would want to be notified before I enter the room", "users to be notified that there is data being collected"). The collection of other datasets including temperature and motion seemed not to be as important.

During the discussion on different kinds of sensing and data collection, different sensors worried participants in different places: the collection of data in a public space (e.g. library) was acceptable and not problematic-though participants noted that such spaces should not collect arbitrarily data ("only collect data that is relevant to their business. Noone should monitor conversations."). We observed that then the participants' understanding of "personally identifiable data" changed based on the context. For example, the collection of sensor data in private spaces (e.g. their homes) appeared to be much more sensitive-even if it was non-identifiable data such as humidity, dust or energy ("embarrassed if it shows lots of dust"). Participants generally would not like anyone to be able to access any sensing data that was collected in a private environment including their home and office.

\subsection{Feedback on the Design Probe}

To evaluate our existing prototype system for creating IoT sensor awareness and display personalisation, we asked specific questions with regards to the usability of the prototype system and asked participants to suggest improvements or changes to the system.

Generally, the access to an overview of the kinds of data that sensors deployed in a space are capable of collecting appeared to be more important to the participants than seeing the actual data that was collected. Participants identified several means of accessing such information, starting initially with integration into a website that informs users about deployed sensors. While participants noted that they would "maybe" or only "once" access such a website, nevertheless "it would be nice to have the option." However, participants pointed out that they wished to be explicitly notified about any data collection in the space they are about to enter if personally identifiable data was collected. Asked about the means for such notifications, participants proposed this could be available by logging into staff or student portals on their computers ("would be on the laptop when you login as part of [a staff portal]"). Other participants mentioned displays or projectors that would typically be located in meeting rooms and other spaces to inform people about personally identifiable data collection-similar to CCTV ("make you aware that you are being recorded.").

While participants seemed more concerned about the kinds of data that are collected (e.g. audio and video), seeing graphs that show the actual sensor readings in real-time made participants more interested. In terms of accessing the actual data, participants seemed more interested in remote data access than local access. For example, access to data was considered useful if it enabled participants to monitor their homes but not to understand more about the sensing in the local environment. Participants responded favourably to the recommendations we communicate to users ("I like the recommendations.").

When asked if participants could imagine interacting with sensor data, e.g. to report sensor faults, we noticed that they generally assumed that sensors would always work properly, or that sensor faults would be automatically detected. Interestingly, none of the participants mentioned the mobile phone as a possible mean of access to sensor data (both for local and remote monitoring).

\section{Displays as Windows to the IoT}

Our design probe illustrates how pervasive displays might improve overall interaction between users and the IoT by providing "windows" onto the respective infrastructure. In the following sections we discuss the opportunities for using pervasive displays as universal IoT interaction points with respect to both overall system design (i.e. how pervasive displays can improve an IoT system) and future research (i.e. challenges that will need to be addressed by the community). We structure our discussion around three key characteristics of pervasive displays: shared visibility, situatedness, and distinct interaction patterns.

\subsection{Shared Visibility}

A defining feature of public and semi-public displays is their shared nature: interactions with displays happen within view of others. Consequently, observing one's interaction with such a display is also somewhat socially accepted, in contrast to, 
say, observing another person's laptop screen or even mobile phone display. This may have important consequences for social interactions in the context of IoT infrastructure control. While mobile interfaces (e.g., mobile phone apps) are perfectly able to offer any level of desired inspection and control capabilities for IoT infrastructures, providing a similar access interface on a public or semi-public display immediately makes the act of interacting with an IoT infrastructure a social experience. Instead of shutting others out like a mobile interaction would do (called "phubbing" [5, 22]), controlling or inspecting a set of IoT devices with the help of a pervasive display both keeps one's current interaction partners informed about one's intentions, and implicitly supports them observing those interactions. In contrast, operating or inspecting an IoT infrastructure through a mobile phone app introduces ambiguity, as one's interaction partners are typically unable to discern why one is withdrawing from a face-to-face social engagement: is one trying to dim the lights in the room, or simply reading chat messages? Pervasive displays thus offer a novel system design opportunity in the form of more socially accepted control interfaces.

However, fixed pervasive displays are clearly unable to provide the convenience of a mobile phone interface, i.e., putting the interface into the user's hands. In order to control or inspect an IoT system with the help of a fixed display, a user typically will need to physically engage with a fixed landmark (i.e., the display) in a space, most likely requiring physical movement. Also, in some situations, e.g., a public presentation, being able to discreetly operate a smart room may be desired in order to not interrupt the speaker. Pervasive displays thus offer a novel research opportunity in the form of understanding what IoT devices should best be controlled on what type of device and in what type of circumstances.

\subsection{Situatedness}

A key difference between a fixed display and a mobile display is the former's situatedness: by virtue of being fixed to a wall or mounted on a desk, the fixed display is in principle able to take its location into account. Situated displays are thus much better able to take a local context into account - in the case of IoT networks, a situated display can easily limit its sensing and actuating controls to local IoT devices only, rather than prompting the user to actively select devices or information from a virtually unbound list of items. Clearly, localization in principle also enables mobile devices to sense their "location context" and thus similarly adapt their interfaces. However, such a dynamic approach inevitably encounters border conditions (e.g., a location between two rooms) that, even assuming perfect localization capabilities, may present the user with unanticipated user interface options. Pervasive displays here offer a novel system design opportunity in the form of "localized" controls.

This is not to say that situatedness will make creating appropriate inspection and control interfaces easy. Clearly, setting up a control interface for even a reasonably small IoT network will still require significant setup costs. Groups of devices must be defined, automation policies created, and sensing overviews laid out. However, by focusing such interfaces on a nearby situated display, these set up costs can be amortised over multiple users. Anybody interacting with the IoT infrastructure through that display will be able to benefit from such a prior setup, without having to configure their own personal access devices (e.g., mobile phones). While localized setups can of course in principle also be distributed to personal devices (e.g., by providing a Web-based interface that people connect to), such setups not only need to take into account a significant variety in device form factors, operating systems, and user preferences, but also will need to solve the "bootstrap" problem of pointing the mobile user to the right interface (e.g., by offering a QR-encoded or NFC-provided URL). A local display on the other hand can be easily adjusted to provide an optimized user experience for a specific local IoT infrastructure. Obviously, a multitude of such situated fixed displays, in particular at larger scale (e.g., a large home or even small office) will make the individual configuration of each displays difficult. In order for pervasive displays to play a large role in IoT infrastructure inspection and control, setup procedures must be simplified and easily be clonable or automatable so that one setup can be replicated and subsequently adjusted for nearby displays. Pervasive displays thus create a novel research opportunity in the form of automatic localized setup procedures.

Beyond the obvious ability of situated interfaces to select the "right" subset of IoT devices to inspect and/or control, situatedness also offers secondary interaction benefits due to their lower suitability towards general, non-situated services: while today's mobile phones are powerful personal computers that, just as our PCs and laptops, allow us to choose from hundreds of installed applications (e.g., read email and web pages, edit documents and drawings, order online, playing games), situated displays are usually offering a much smaller selection of applications. While also being a general purpose computer, it simply makes less sense to have such a large number of applications available on a pervasive display - either for security (being public resources) or lack of demand (few people may want to read their emails on such devices). As a result, situated displays can offer a much more focused selection of applications, thus greatly simplifying one's interaction with a local IoT infrastructure. Instead of having to browse through hundreds of installed apps on one's mobile phone, one simply chooses from a handful of supported apps on a single menu page or application bar. Pervasive displays hence offer a novel system design opportunity in the form of simpler and thus faster interactions.

At the same time, this situatedness also makes it challenging to decide where the local/remote boundary is actually located. Does it make sense for a display situated in the living room to also control IoT infrastructure for the rest of the house? Pervasive displays thus create a novel research opportunity in the form of understanding user preferences and mental models when it comes to operating IoT devices from afar. 


\subsection{Distinct Interaction Characteristics}

Fixed pervasive displays entail a set of distinct interaction characteristics that may provide immediate benefits to inspecting and/or controlling IoT infrastructures, predominantly due to their size, but also due to their shared (non-personal) nature.

\subsubsection{Fewer Task Distractions}

Having fewer applications on a system (see "Situatedness" above) not only makes it easier to find and launch the desired interface, but also reduces the chance for task interruptions. Personal notifications ("You got mail!" "You have 12 new posts on your wall!") provide instant gratification and are difficult to not follow-up [10]. It is not uncommon to get out a smartphone in order to quickly check the time, only to find oneself reading friends's recent Facebook posts. A pervasive display-based interface to control local IoT systems thus offers a novel system design opportunity by virtue of having fewer personal applications (e.g., email, chat, social media) and hence being less distracting during operation. The challenge will obviously be to strike an apropriate balance between limiting display applications in order to simplify operation and eliminate distractions, and offering a rich set of useful services. In principle, large displays could easily support userappropriation as secondary screens, e.g., similar to the Google Chromecast system that allows compatible applications to simply stream out content to a connected display. Pervasive displays thus create a novel research opportunity in the form of understanding how to define this balance.

\subsubsection{Large Screen Real-Estate}

Even with the latest trends of smart phones to become bigger and bigger ("phablets"), a mobile interface will almost always have less screen real-estate available than a fixed pervasive display. This allows interfaces to not only be more expressive, e.g., when providing inspection tools, but also limit the number of screens needed to lay out a more complex interface. Pervasive displays hence offer a novel system design opportunity in the form of more expressive interfaces.

However, this is not to say that such interfaces will be easier to construct - there is probably a just as large variety of screen sizes and interaction methods for pervasive displays that will make it a challenge to play out a suitable interface for a particular type of display, in particular if one does not want to handcraft the interface of every single display. Pervasive displays thus create a novel research opportunity in the form of how to automatically create such interfaces.

\subsubsection{Supporting Ambient Notification}

Last but not least, fixed pervasive displays also make it easier to gather awareness of an IoT system in an ambient fashion. Instead of having to actively get out a personal display device (i.e., a mobile phone or a smart watch), a visible display allows one to capture important system states in passing. At the same time, given suitably subdued interface elements and/or brightness settings, such information can easily be ignored. Having a readily-available yet unobtrusive view into an IoT-system's state may improve user understanding and acceptance. Pervasive displays hence offer a novel system design opportunity in the form of providing glanceable ambient information. At the same time, pervasive displays create a novel research opportunity in the form of designing such unobtrusive yet informative displays.

\section{Related Work}

\subsection{IoT in Public Spaces}

In recent years we have witnessed an explosion in research and commercial development activities in relation to the IoT. Numerous platforms have been developed to capture and visualise IoT data, including ThingWorx [21], IBM Bluemix [12], the Intel IoT platform [13], and DGLogik for IoT automation [8]. Significant research has been conducted into IoT sensing in public spaces. Large scale urban deployments such as [26] have been created to demonstrate potential applications of the IoT, and national governments have launched strategic initiatives (e.g. https://iotuk.org.uk). Open Bristol is an example for an initiative that collects large amounts of IoT sensor data regarding "many aspects of city life, including energy, air quality and traffic flows" [2] and makes these available through an open platform.

\subsection{Privacy and Awareness of Data Capture}

Early work has already described and raised concerns regarding the deployment of ubiquitous systems in the real-world and the implications for users' privacy [14]. To address this issue, Langheinrich has developed "a privacy awareness system for ubiquitous computing environments" [14] targeting the collectors of data and allowing them to describe and announce the details about the levels of data collection. In addition, the system provides details to users track and withdraw the data they have implicitly or explicitly contributed to the system [14]. More recently, Shaw et al. developed a prototype system that notifies users utilising smart watches about physical areas with a certain level of IoT sensing capabilities and data collection and displays the with the space associated data sharing policies [24]. The system is designed to provide a level of transparency for the user and make them aware of potentially personal data collection in a space-increasing the trust of the user into pervasive environments and allowing the user to choose whether or not to contribute to the data collection [24]. Researchers have also explored ways that allowed users to express their preferences regarding the data collection and sharing. Myles, Friday, and Davies developed a system that allows users to express complex rules and policies regarding the disclosure of their personal data to the system and thirdparties [19]. Specifically addressing the data collection in the user's personal context (e.g. in their homes), Mun et al. developed "Personal Data Vaults"- ensuring that the ownership and 
control about the data remains with the user, and providing the user with the ability to control access to their data through a pre-defined set of data policies [18].

\subsection{Public Information Visualisation}

Information visualisation is traditionally aimed at specific domain experts who have the knowledge and skills to interpret the data in order to derive information and knowledge [4]. However, in recent years an increasing number of data visualisations have been designed for non-expert users [20]. These more 'casual' information visualisations can help communicate changes in data to a broader audience. By using 'ambient' visualisations that reside in the periphery of users, we can represent information visualisation in public spaces for people who are interested and curious [20]. Early examples of such visualisations include the "Information Percolator" [11], in which the authors have integrated the visualisation into a "aesthetically pleasing decorative object" [11]. In this particular example, the display is formed by a row of tubes that are filled with water. Information is displayed through air bubbles that are automatically released by the system and support the visualisation of binary images. In more recent work, Mirlacher et al. specifically looked at novel ways of displaying information in public spaces through ambient and embedded displays [16]. The authors used a set of LEDs integrated into a Nabaztag rabbit as a visualisation component to the user, and used the prototype to investigate the feasibility of highly embedded displays for information dissemination in public spaces. In a related approach, Rushikesh and Sivappagari used small-scale displays mounted directly onto the sensing device to show its current pollution values [23]. Folea and Mois developed an integrated sensor and display to monitor and visualise the air quality of the work environment [9]. The sensing device collects humidity, pressure and brightness levels and transmits these through wireless communication protocols to a server that makes the collected datasets available for further analysis.

\section{Conclusions}

IoT devices are becoming ever more pervasive, leading to "smart spaces" and the potential to collect detailed physical analytics. However, much of this smartness is made deliberately invisible to the user - echoing Weiser's vision of calm computing and technology that fades into the background. The resulting lack of user awareness and can lead to poor user experience/frustration, mistrust, suspicion, inability to capitalise on benefits and security vulnerabilities. The use of Pervasive Display within IoT infrastructure offer intriguing possibilities to support increased user awareness due to their shared visibility (better social acceptance for operating), situatedness (easierto-use localized controls), and distinct interaction patterns (less distractions, lower UI complexity, better support for ambient notifications). At the same time, integration of pervasive displays with the IoT opens up a wealth of novel research opportunities: what does display control and display analytics look like in an IoT context? how do we best capitalize on the distinct UI capabilities of Pervasive Displays in the context of IoT infrastructures? and how can we automate the integration of Pervasive Displays in an IoT network and enable it as a locus of control and inspection?

We hope that our article offers a first step towards a better integration of Pervasive Displays into the IoT eco-system, and points to a fruitful intersection of these two strands of research.

\section{Acknowledgments}

This work has been partially funded by the UK EPSRC as part of "PETRAS IoT Research Hub - Cybersecurity of the Internet of Things" (EP/N023234/1) and "PACTMAN: Trust, Privacy and Consent in Future Pervasive Environments" (EP/N028228/1), and was made possible with the support of a Google Faculty Award.

\section{References}

[1] Mara Balestrini et al. "IoT community technologies: leaving users to their own devices or orchestration of engagement?" In: EAI Endorsed Transactions on Internet of Things 1.1 (2015).

[2] Bristol is Open Ltd. Smart City Research and Development Platform. 2018. URL: https : / / www . bristolisopen.com (visited on 02/03/2017).

[3] Andrea Caragliu, Chiara Del Bo, and Peter Nijkamp. "Smart cities in Europe". In: Journal of urban technology 18.2 (2011), pp. 65-82.

[4] Stuart K Card, Jock D Mackinlay, and Ben Shneiderman. Readings in information visualization: using vision to think. Morgan Kaufmann, 1999.

[5] Varoth Chotpitayasunondh and Karen M. Douglas. "How "phubbing" becomes the norm: The antecedents and consequences of snubbing via smartphone". In: Computers in Human Behavior 63 (Oct. 2016), pp. 918. ISSN: 07475632. DOI: $10.1016 / \mathrm{j}$. chb . 2016 . 05.018 URL: http : / / linkinghub . elsevier . com / retrieve/pii/S0747563216303454.

[6] Sarah Clinch et al. "Yarely: A Software Player for Open Pervasive Display Networks". In: Proceedings of the 2Nd ACM International Symposium on Pervasive Displays. PerDis '13. Mountain View, California: ACM, 2013, pp. 25-30. ISBN: 978-1-4503-2096-2. DOI: 10 . 1145/2491568 . 2491575, URL: http://doi . acm . org/ $10.1145 / 2491568.2491575$ 
[7] Nigel Davies et al. "Personalisation and Privacy in Future Pervasive Display Networks". In: Proceedings of the 32Nd Annual ACM Conference on Human Factors in Computing Systems. CHI '14. Toronto, Ontario, Canada: ACM, 2014, pp. 2357-2366. ISBN: 978-14503-2473-1. DOI: $10.1145 / 2556288$. 2557287. URL: http://doi.acm.org/10.1145/2556288.2557287

[8] DGLogik. Modern IoE Application Platform. 2017. URL: http : / / www . dglogik . com/ (visited on 02/03/2017).

[9] Silviu C. Folea and George Mois. "A Low-Power Wireless Sensor for Online Ambient Monitoring”. In: IEEE Sensors Journal 15.2 (Feb. 2015), pp. 742-749. ISSN: 1530-437X. DOI: 10.1109/JSEN. 2014.2351420

[10] Benjamin V. Hanrahan and Manuel A. Pérez-Quiñones. "Lost in Email: Pulling Users Down a Path of Interaction". In: Proceedings of the 33rd Annual ACM Conference on Human Factors in Computing Systems - CHI '15. New York, New York, USA: ACM Press, 2015, pp. 3981-3984. ISBN: 9781450331456. DOI: 10. 1145/2702123.2702351. URL: http://dl . acm . org/ citation. cfm?doid=2702123.2702351

[11] Jeremy M Heiner, Scott E Hudson, and Kenichiro Tanaka. "The information percolator: ambient information display in a decorative object". In: Proceedings of the 12th annual ACM symposium on User interface software and technology. ACM. 1999, pp. 141-148.

[12] IBM. Bluemix. 2017. URL: https : / / www . ibm . com/ cloud-computing/bluemix/internet-of-things (visited on 02/03/2017).

[13] Intel. Intel IoT Platform. 2017. URL: http : / / www . intel . com / content / www / us / en / internet - of things/infographics/iot-platform-infographic . html (visited on 02/03/2017).

[14] Marc Langheinrich. "A Privacy Awareness System for Ubiquitous Computing Environments". In: UbiComp 2002: Ubiquitous Computing: 4th International Conference Göteborg, Sweden, September $29-$ October 1, 2002 Proceedings. Ed. by Gaetano Borriello and Lars Erik Holmquist. Berlin, Heidelberg: Springer Berlin Heidelberg, 2002, pp. 237-245. ISBN: 978-3540-45809-8. DOI: $10.1007 / 3-540-45809-3$ _19. URL: https://doi.org/10.1007/3-540-45809-3_19.

[15] Donald McMillan et al. "Data and the City". In: Proceedings of the 2016 CHI Conference on Human Factors in Computing Systems. ACM. 2016, pp. 29332944.

[16] Thomas Mirlacher et al. "Ambient rabbits likeability of embodied ambient displays". In: European Conference on Ambient Intelligence. Springer. 2009, pp. 164-173.

[17] Klaus Moessner. IoT not only as smart city enabler but as research enabler for other disciplines. Tech. rep. 6th Japan-EU Symposium on ICT Research and Innovation, Oct. 2016.
[18] Min Mun et al. "Personal data vaults: a locus of control for personal data streams". In: Proceedings of the 6th International Conference on Emerging Networking Experiments and Technologies. ACM. 2010, p. 17.

[19] Ginger Myles, Adrian Friday, and Nigel Davies. "Preserving privacy in environments with location-based applications". In: IEEE Pervasive Computing 2.1 (2003), pp. 56-64.

[20] Zachary Pousman, John Stasko, and Michael Mateas. "Casual information visualization: Depictions of data in everyday life". In: IEEE transactions on visualization and computer graphics 13.6 (2007).

[21] PTC. ThingWorx. 2017. URL: https://www. thingworx . com (visited on 02/03/2017).

[22] James A. Roberts and Meredith E. David. "My life has become a major distraction from my cell phone: Partner phubbing and relationship satisfaction among romantic partners". In: Computers in Human Behavior 54 (Jan. 2016), pp. 134-141. ISSN: 07475632. DOI: $10.1016 /$ j . chb . 2015 . 07 . 058 URL: http : / / linkinghub . elsevier.com/retrieve/pii/S0747563215300704

[23] R. Rushikesh and C. M. R. Sivappagari. "Development of IoT based vehicular pollution monitoring system". In: 2015 International Conference on Green Computing and Internet of Things (ICGCIoT). Oct. 2015, pp. 779783. DOI: 10.1109/ICGCIoT . 2015.7380568.

[24] Peter Shaw et al. "Using Smartwatches for Privacy Awareness in Pervasive Environments". In: Poster at the 18th International Workshop on Mobile Computing Systems and Applications. 2017.

[25] Noam Shoval. "Tracking technologies and urban analysis". In: Cities 25.1 (2008), pp. 21-28.

[26] Smart Santander consortium. Smart Santander. 2017. URL: http : / / www . smartsantander . eu (visited on 02/03/2017). 